\title{
Achilles tendon healing in rats is improved by intermittent mechanical loading during the inflammatory phase
}

Therese Andersson, Pernilla Eliasson and Per Aspenberg

\section{Linköping University Post Print}

N.B.: When citing this work, cite the original article.

This is the pre-reviewed version of the following article:

Therese Andersson, Pernilla Eliasson and Per Aspenberg, Achilles tendon healing in rats is improved by intermittent mechanical loading during the inflammatory phase, 2011, Journal of Orthopaedic Research, (30), 2, 274-279.

which has been published in final form at:

http://dx.doi.org/10.1002/jor.21511

Copyright: Wiley-Blackwell http://eu.wiley.com/WileyCDA/Brand/id-35.html

Postprint available at: Linköping University Electronic Press http://urn.kb.se/resolve?urn=urn:nbn:se:liu:diva-70772 
Achilles tendon healing in rats is improved by intermittent mechanical loading during the inflammatory phase

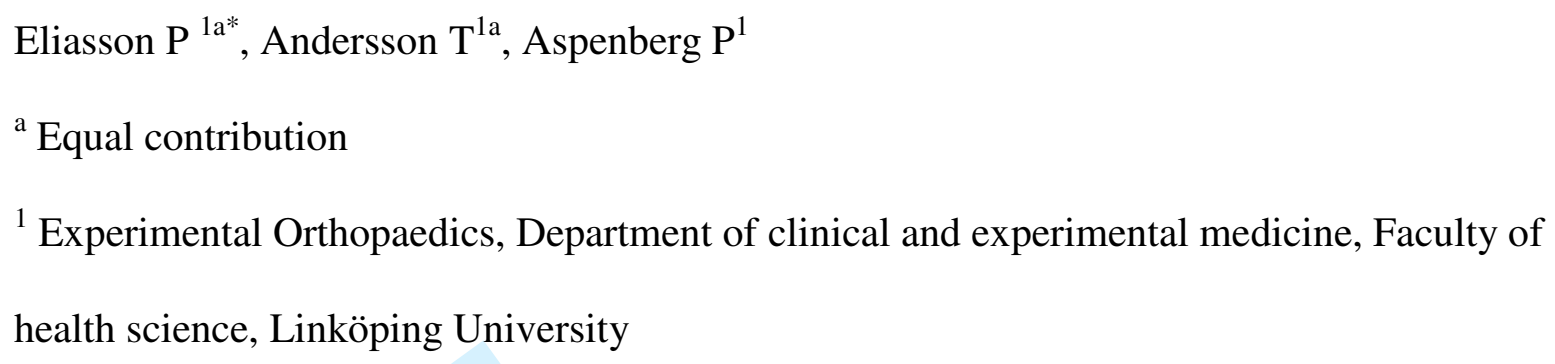

Running title: Tendon healing and early loading 


\begin{abstract}
Tendons adapt to changes in mechanical loading, and numerous animal studies show that immobilization of a healing tendon is detrimental to the healing process. The present study addresses whether the effects of a few episodes of mechanical loading is different during different phases of healing. Fifty female rats underwent Achilles tendon transection, and their hind limbs were unloaded by tail suspension on the day after surgery. One group of 10 rats was taken down from suspension to run on a treadmill for 30 minutes per day, on days 2-5 after transection. They were euthanized on day 8. Another group underwent similar treadmill running on days 8-11 and was euthanized on day 14. Completely unloaded groups were euthanized on day 8 and 14. Tendon specimens were then evaluated mechanically. The results showed that just 4 loading episodes increased the strength of the healing tendon. This was evident irrespective of the time-point when loading was applied (early or late). The positive effect on early healing was unexpected, considering that the mechanical stimulation was applied during the inflammatory phase, when the calluses were small and fragile. A histological study of additional groups with early loading also showed some increased bleeding in the loaded calluses. Our results indicate that a small amount of early loading may improve the outcome of tendon healing. This could be of interest to clinical practice.
\end{abstract}

\title{
KEYWORDS
}

Early loading, tail-suspension, unloading, mechanical testing, cell differentiation

\section{INTRODUCTION}

The influence of early mobilization on the outcome of Achilles tendon healing has attracted increasing attention. New rehabilitation programs recently tested include early weight bearing $(1,2)$ and the use of orthoses, designed to permit certain ankle movement and some weight 
bearing $(3,4)$. The results from these studies indicate, to some extent, that early mobilization and weight bearing have a positive effect on the healing process. However, clinical research has so far not been able to distinguish between the effects of motion on the readaptation of periarticular tissues and the effects of traction on tendon healing. It is unknown to what extent weight bearing on the injured leg translates into traction forces in the healing Achilles tendon. There is also probably a risk of complications, especially re-rupture, if loading is applied too early. The improved clinical results, however, are in line with previous studies of the effects of mechanical loading in animal models and on tendon cells in vitro. Cyclic loading regimes up-regulate both the gene-expression and production of collagen by tendon fibroblasts in vitro $(5,6)$. This increase in collagen expression can also be seen in intact animal tendons, subjected to increased loading $(7,8)$. In fact there are many studies showing that intact tendons adapt to changes in mechanical loading (9-14), and numerous animal studies show that immobilization of a healing tendon is detrimental for the healing process (15-20).

To gain better understanding regarding the effect of mechanical loading on tendon healing, we have previously studied the effects of short daily loading episodes on otherwise unloaded healing Achilles tendons in rats (17). The results showed that 15 minutes of daily mechanical loading, from the third day after tendon transection, was sufficient to increase callus thickness and strength of the healing tendons at 14 days. There was only a weak correlation between the duration of the loading episode (15, 30 or 60 minutes) and the effect on healing. Moreover, an additional daily loading episode for 15 minutes, 8 hours after the first one, did not further improve healing. This indicated that the response to a loading episode lasted for several hours, and couldn't be much boosted by more of the same type of loading the same day. These results raised the question how many times a short loading episode has to be repeated to improve tendon healing in otherwise unloaded tendons. 
In another model for unloaded tendon healing in rats, mechanical loading had different effects on the gene expression pattern depending on the phase of tendon healing (21). At three days of healing (during the inflammatory phase), gene expression of IL- $1 \beta$, TNF- $\alpha$ and TGF- $\beta$ was decreased in loaded tendon calluses compared to calluses unloaded by Botox injections. Also, procollagen I and III were less expressed in the loaded samples at that timepoint. However, at 14 days of healing (during the proliferative phase), procollagen I and several tendon specific genes were all more expressed in the loaded samples compared to the unloaded. These results suggested strong effects of loading also during the early inflammatory phase of healing, while it is often thought that loading mainly influences matrix formation and remodelling which occur later. The results raised the question if mechanical loading has different effects on tissue strength when applied during different phases of healing.

Having previously studied daily loading episodes for 11 days (days 3-13), we now investigated if even as little as 4 loading episodes could improve the outcome of healing. We also compared the effects of loading during the early, inflammatory phase of healing with loading during the somewhat later proliferative phase. We had two primary hypotheses. First, that 4 loading episodes are sufficient to stimulate healing. Second, that loading has less positive effect during the early inflammatory phase compared to a later proliferative phase of healing. We used tail suspended rats, to allow short and controlled episodes of loading of the otherwise load-protected Achilles tendon. Tail suspension reduces maximal tension forces in the Achilles tendon while maintaining full motion. Because no ground force is applied, the only resistance to plantar flexion is the moment produced by the weaker dorsal flexors, with their shorter lever arm. Even with maximal contraction of these muscles, the calf muscles will be able to balance this moment with minimal effort. The suspended rats were either completely unloaded, 24 hours a day, or allowed daily running periods of 30 min during a 4 
days period. We then compared tendon mechanical properties 3 days after the final loading episode.

\section{MATERIALS AND METHODS}

The experiment was approved by the regional animal ethics committee for animal experiments and institutional guidelines for care and treatment of laboratory animals were adhered to.

Surgery: Fifty female Sprague-Dawley rats, 207 (SD 14) g, were used in this experiment, 40 rats for mechanical, and 10 for histological evaluation. All rats were habituated to a treadmill apparatus $(12 \mathrm{~m} / \mathrm{min})$, before the experiment started. Since rats are active during the night, the 12 hour light and dark cycle was switched, so that handling of the animals was done during their active period. On the day of surgery, the rats were anesthetized with isoflurane gas (Forene, Abbot Scandinavia, Solna, Sweden) and given antibiotics ( $25 \mathrm{mg} / \mathrm{kg}$, Oxytetracycline, Engemycin; Intervet, Boxmeer, Holland), and analgesics $(0.045 \mathrm{mg} / \mathrm{kg}$, Buprenorphine, Temgesic; Schering-Plough, Brussels, Belgium) preoperatively. The skin on the right hind limb was shaved and washed with chlorhexidine ethanol, and the surgery was performed under aseptic conditions. A transverse incision was made in the skin lateral to the right Achilles tendon and the Achilles-plantaris tendon complex was exposed. The Achilles tendon was transected transversely approximately $3 \mathrm{~mm}$ from the calcaneal bone, and a $3 \mathrm{~mm}$ segment was removed. Thereafter the tendon was left to heal unsutured. The plantaris tendon was completely removed to avoid interference during healing and mechanical testing.

Unloading: The day after surgery, all 50 rats were subjected to hindlimb unloading by tail suspension. The tail suspension was carried out in special cages with an overhead system, allowing the rats to rotate and move in all directions. The rats were kept one per cage and had been acclimatized to the suspension cages before surgery. For suspension, an adhesive tape was attached to the rat's tail. The tape was connected to the overhead system by a fish-line 
swivel and a fish line. The hind limbs were lifted just above the cage floor during the entire experiment and the rats moved around on their fore limbs, as described more in detail elsewhere (22). The rats were housed in a room with temperature maintained at $24^{\circ} \mathrm{C}$ and were given food and water ad libitium.

Experimental set-up: The study consisted of two parts, one with biomechanical and one with histological evaluation.

Biomechanical experiment: After unloading, 40 rats were randomized into four groups by lottery. Two groups were completely unloaded for 8 or 14 days (early and late control group) and two groups were subjected to exercise. The exercise groups were let down from the suspension to run on the treadmill for 30 minutes per day $(9 \mathrm{~m} / \mathrm{min}$, slightly uphill) on day 25 (early exercise group) or on day 8-11 (late exercise group, fig 1) (17). The animals were monitored during the entire exercise to ensure that loading was applied to the injured limb. The animals in the two exercise groups were completely unloaded for 72 hours following the final exercise episode (day 5 or 11), before they were sacrificed together with the corresponding unloaded control animals on day 8 or 14 .

Histological experiment: The histological experiment was performed to evaluate if early weight bearing during the inflammatory phase caused bleeding or other signs of injury due to the loading applied on the immature callus tissue. Ten rats were included in the histological experiment. After unloading, the rats were randomized into two groups, one group was completely unloaded for 5 days and one group was subjected to exercise by letting the rats down from the suspension to run on the treadmill for 30 minutes per day $(9 \mathrm{~m} / \mathrm{min}$, slightly uphill) on day 2-5. The amount of loading corresponded to the loading applied in the early exercise group in the biomechanical experiment. The animals in the exercise group were euthanized one hour after finishing the final loading episode together with the corresponding unloaded control group. 
Mechanical evaluation: On the day of sacrifice (after 8 or 14 days) the animals were anaesthetized with a subcutaneous injection of dexmedetomidine $(0.5 \mathrm{mg} / \mathrm{kg}$, Dexdomitor; Orion Pharma, Esbo, Finland) and ketamine (75 mg/kg, Ketaminol; Intervet, Boxmeer, Holland), while still suspended, and then killed by an overdose of pentobarbital sodium (APL, Stockholm, Sweden). The right Achilles tendon together with the calcaneal bone and parts of the gastrocnemius and soleus muscle complex were harvested. Sagittal and transverse diameters of the midpart of the callus tissue were measured with a slide caliper and crosssectional area was calculated by assuming an elliptical geometry. The old tendon stumps were visualized by transillumination of the entire callus, and the distance between them was measured and referred to as the gap distance. For mechanical testing, the muscles were carefully scraped of the tendon fibers and fine sand paper was used to fix the fibers in a metal clamp. The distance between the metal clamp and the calcaneal bone was used as an approximation of the tendon length. The calcaneal bone was fixated in a custom-made clamp in $30^{\circ}$ dorsiflexion relative to the direction of traction. The tendon was mounted vertically via the metal clamp in the materials testing machine (100R, DDL Inc., Eden Praire, USA) and pulled at constant speed $(0.1 \mathrm{~mm} / \mathrm{s})$ until failure. Force at failure (i.e. peak force) and stiffness were calculated by the software of the testing machine. The investigator marked a linear portion of the elastic phase of the curve for stiffness calculation. Peak stress and an estimate for elastic modulus were calculated afterwards. The estimate for elastic modulus was based on assumptions of tissue homogeneity and that the entire specimen was shaped as an elliptic cylinder. It is likely to differ considerably from the modulus of the callus tissue in the gap, but still allow group comparisons. All measurements and calculations were carried out by an investigator blinded for treatment and group belonging of the specimens.

Histological evaluation: The rats were euthanized one hour after completing the final exercise on day 5. The animals from the corresponding unloaded control group were 
sacrificed at the same time point. Before euthanasia, the rats were anaesthetized with a subcutaneous injection of dexmedetomidine $(0.5 \mathrm{mg} / \mathrm{kg})$ and ketamine $(75 \mathrm{mg} / \mathrm{kg})$, while still suspended, and then killed by an overdose of pentobarbital sodium. The hind limbs were kept unloaded throughout the whole procedure. The right Achilles tendon callus was harvested together with a small piece of the calf muscle (for orientation of the tissue) and fixed in $4 \%$ phosphate buffered formaldehyde. After dehydration, the specimens were embedded in paraffin and sectioned parallel to the longitudinal axis of the tendon. One slide per specimen, comprising the full length of the tendon callus, was stained with Ehrlich Hematoxylin and Eosin. The slides were analyzed in a light microscope to estimate the extent of bleeding in each specimen, as a sign of iterated trauma. Each specimen was examined by two independent investigators without any fixed criteria other than to estimate the occurrence of bleeding. Bleeding was defined as large accumulations of erythrocytes in the tissue. Both investigators independently graded the samples as either 0 or 1 , where 1 corresponded to extensive bleeding. One of the investigators classified one specimen as in-between (0.5). The investigators were blinded for treatment during the evaluation.

Statistics: The mechanical results were analyzed by a two-way Anova with time and loading status as independent variables. Peak force was the primary outcome variable. According to plan, we first tested the hypothesis that 4 loading episodes had an influence on healing, thereafter that there was an interaction between time and loading status. Having found no such interaction, we formulated the secondary hypothesis that early loading would have an effect on strength, and tested this by Student's t-test, and finally did the same for later loading. The rats were randomized into groups by lottery after surgery and all measurements and calculations were performed by an investigator unaware of previous treatment. 


\section{RESULTS}

Mechanical evaluation: A two-way Anova of all groups revealed that the 4 loading episodes improved the mechanical properties of the tendons, with an increased peak force and stiffness $(\mathrm{p}<0.0001$ for both). The peak stress in the healing tendons was also improved $(\mathrm{p}=0.006)$ as well as the energy uptake $(\mathrm{p}=0.002)$. All these four variables were also increased with time $(\mathrm{p} \leq 0.002$ for all). Elastic modulus was increased with time $(\mathrm{p}=0.04)$, but was not significantly affected by loading.

After 8 days of healing, the peak force was $60 \%$ higher in the exercise group compared to the corresponding controls ( $\mathrm{p}=0.01$, figure $2 \mathrm{~A}$, table 1 ). In order to confirm the statistical robustness of this finding, we also made a Mann-Whitney test $(\mathrm{p}=0.007)$.

The stiffness was $30 \%$ higher in the exercise group $(\mathrm{p}=0.05$, table 1$)$. Peak stress, a material property, was $40 \%$ higher in the early exercise group compared to the controls $(p=0.03$, figure $2 b$ ). Elastic modulus was not significantly affected.

After 14 days of healing, the pattern of the results was similar to the early time point but with higher absolute values. The peak force was improved by $50 \%$ after late exercise compared to the corresponding controls $(\mathrm{p}=0.006$, figure 2 , table 1$)$ and the stiffness was $30 \%$ higher $(\mathrm{p}=0.01$, table 1$)$. Peak stress increased by $40 \%(\mathrm{p}=0.051)$ compared to the control group.

The cross-sectional area did not differ between the unloaded controls and exercise groups at any time point (table 1). There was also no difference in cross-sectional area between 8 and 14 days. The gap distance was also unaffected by loading at both time-points.

One rat in the early control group, one rat in the late exercise group, and two rats in the early exercise group were excluded from the experiment because the suspension device on one occasion loosened from the tail, resulting in unwanted loading (table 1). 
Histological evaluation: Signs of bleeding were found in all samples irrespective of loading (fig. 3a, b). However, the regions with bleeding were more extensive in the exercise group compared to the completely unloaded group (table 2).

The specimens were also divided into two groups (suspected exercise or suspected control) based on the amount of bleeding. The two investigators, blinded for treatment, separately came to the same results, which agreed completely with actual treatment. Larger amounts of erythrocytes in the tissue were characteristic for the exercise group, whereas less bleeding was rare but never absent in the unloaded control samples.

\section{DISCUSSION}

A few, short episodes of loading could improve the strength of the tendon callus, both when applied during the early inflammatory phase or the later, proliferative phase of healing. A 50$60 \%$ increase in peak force was found in both exercise groups compared to their unloaded controls. The exercise applied during the early phase gave the tissue the same strength at 8 days as the unloaded controls at 14 days. This positive effect on early healing was unexpected, considering that the mechanical stimulation was applied during the inflammatory phase. At this time there is only a fragile callus at the healing site, and loading might cause damage. Indeed, the tissue showed histological signs of repeated damage in the form of increased bleeding. The fact that this added damage was not detrimental could be associated with the rest periods between the short loading episodes, allowing trauma-boosted repair mechanisms to work. The results might also be related to our previous finding that the expression of some inflammation-related genes, for example IL-1 and TNF- $\alpha$, are reduced by mechanical loading in the early phase of healing (21). There are, however, a number of differences between these two studies. The animals in the previous study were either unloaded or loaded the whole time, whereas in the present study loading was only applied for 30 minutes per day. 
Although it might be unclear if reduction of inflammatory mediators should be expected to be good or bad for healing, it has been shown that inhibition of IL-1 receptor signalling reduces the deterioration caused by stress-shielding in intact patellar tendons (23). These results and our previous gene expression data (21) together suggest that IL-1 might play an important role for the detrimental effect of unloading in tendons.

In clinical practice, loading during the inflammatory phase is considered to be detrimental. It has also previously been shown that tenotomized and sutured Achilles tendons in rats become stronger at day 8 if immobilized during days 1-5, compared to tendons immobilized days 1-2 and 5-8, suggesting that loading is detrimental specifically during days 1-5 in this model (16). However, the main difference between that study and ours is that we allowed loading only for short periods of time each day. The positive effect of short isolated loading episodes might depend on sufficient time for the tissue to recover and respond before an additional episode is applied. Also, despite loading, there was no elongation of the callus in our experiment. In previous experiments with this model (17), we have even seen a significant shortening of the tendon gap after daily 30 minutes loading episodes for 11 days. This suggests that early loading might be more beneficial than previously thought, if only sufficient periods for unloaded recovery are allowed.

The positive effect of loading on mechanical properties during the later phase of healing was more expected. These findings agree with the generally accepted positive effects of mobilization and weight bearing during the later phase of healing. Proliferating and remodeling tissue is thought to contain a larger proportion of cells able to respond to mechanical stimuli, compared to the granulation tissue of early healing. Still, it is interesting that as little as 4 short episodes of exercise were enough to improve strength. In this study, tissue quality (i. e. peak stress) was improved by mechanical stimulation. We have not seen this before; previous experiments using this model have mainly shown an 
increased cross-sectional area, leading to increased force at failure and stiffness (17). We have generally noted, also in other rat models (21), that loading increased the amount of tissue and thereby the ultimate strength, but not the tissue quality. The explanation for the discrepancy with previous findings might be that we now, contrary to previous studies, included a 3 days recovery period before evaluation. Thus, the healing tissue seems to respond to loading first by proliferating and producing more extra cellular matrix of the same kind, whereas improvement of tissue quality comes as a later response, which perhaps requires that loading is discontinued. This interpretation is built only on biomechanical results. A gene expression analysis might show a switch from a proliferative to a remodeling response after a certain time.

The results indicate that short episodes of loading have a positive effect on tendon healing, suggesting that patients may be allowed short loading episodes following e.g. an Achilles tendon rupture at an early stage. However, this study has several limitations. Animal models are different from the clinical situation. Rats are quadrupeds and can better choose how much loading they put on an injured leg. The rats also had healthy tendons cut transversely, whereas in humans the ruptured tendons often have frayed ends and a history of degeneration. The distance between the tendon stumps in this model is a few millimeters, which probably corresponds to the situation in a human Achilles tendon, at least with nonoperative treatment. Our model could perhaps be seen as a model of one of many fringes in a ruptured human Achilles tendon. Another weakness is that we used only female rats. Tendon response to mechanical loading in humans has recently been shown to depend on sex (24). Unfortunately, the tail suspension model is not suitable for male rats because of their rapid continuous growth. A final weakness of our study is that the positive effect on early healing was not specifically hypothesized in advance. Still, we find it a notable observation. 


\section{ACKNOWLEDGEMENTS}

We thank Bibbi Mårdh for assistance with the histology preparations. We also thank Mats Christensson for manufacturing the tail-suspension cages. The study was supported by unrestricted grants from the Swedish National Centre for Research in Sports, the Swedish Research Council (VR 2009-6725) and the Östergötland County Council.

\section{REFERENCES}

1. Suchak AA, Bostick GP, Beaupre LA, et al. 2008. The influence of early weight-bearing compared with non-weight-bearing after surgical repair of the Achilles tendon. J Bone Joint Surg Am 90:1876-83.

2. Yotsumoto T, Miyamoto W, Uchio Y. Novel approach to repair of acute achilles tendon rupture: early recovery without postoperative fixation or orthosis. Am J Sports Med 38:28792.

3. Mortensen HM, Skov O, Jensen PE. 1999. Early motion of the ankle after operative treatment of a rupture of the Achilles tendon. A prospective, randomized clinical and radiographic study. J Bone Joint Surg Am 81:983-90.

4. Majewski M, Schaeren S, Kohlhaas U, Ochsner PE. 2008. Postoperative rehabilitation after percutaneous Achilles tendon repair: early functional therapy versus cast immobilization. Disabil Rehabil 30:1726-32. 
5. Maeda E, Shelton JC, Bader DL, Lee DA. 2009. Differential regulation of gene expression in isolated tendon fascicles exposed to cyclic tensile strain in vitro. J Appl Physiol 106:50612.

6. Yang G, Crawford RC, Wang JH. 2004. Proliferation and collagen production of human patellar tendon fibroblasts in response to cyclic uniaxial stretching in serum-free conditions. J Biomech 37:1543-50.

7. Heinemeier KM, Olesen JL, Haddad F, et al. 2007. Expression of collagen and related growth factors in rat tendon and skeletal muscle in response to specific contraction types. J Physiol 582:1303-16.

8. Olesen JL, Heinemeier KM, Haddad F, et al. 2006. Expression of insulin-like growth factor I, insulin-like growth factor binding proteins, and collagen mRNA in mechanically loaded plantaris tendon. J Appl Physiol 101:183-8.

9. Olesen JL, Heinemeier KM, Gemmer C, et al. 2007. Exercise-dependent IGF-I, IGFBPs, and type I collagen changes in human peritendinous connective tissue determined by microdialysis. J Appl Physiol 102:214-20.

10. Almeida-Silveira MI, Lambertz D, Perot C, Goubel F. 2000. Changes in stiffness induced by hindlimb suspension in rat Achilles tendon. Eur J Appl Physiol 81:252-7.

11. Eliasson P, Fahlgren A, Pasternak B, Aspenberg P. 2007. Unloaded rat Achilles tendons continue to grow, but lose viscoelasticity. J Appl Physiol 103:459-63.

12. Matsumoto F, Trudel G, Uhthoff HK, Backman DS. 2003. Mechanical effects of immobilization on the Achilles' tendon. Arch Phys Med Rehabil 84:662-7.

13. Reeves ND, Maganaris CN, Ferretti G, Narici MV. 2005. Influence of 90-day simulated microgravity on human tendon mechanical properties and the effect of resistive countermeasures. J Appl Physiol 98:2278-86. 
14. Shin D, Finni T, Ahn S, et al. 2008. Effect of chronic unloading and rehabilitation on human Achilles tendon properties: a velocity-encoded phase-contrast MRI study. J Appl Physiol 105:1179-86.

15. Enwemeka CS. 1992. Functional loading augments the initial tensile strength and energy absorption capacity of regenerating rabbit Achilles tendons. Am J Phys Med Rehabil 71:31-8. 16. Enwemeka CS, Spielholz NI, Nelson AJ. 1988. The effect of early functional activities on experimentally tenotomized Achilles tendons in rats. Am J Phys Med Rehabil 67:264-9. 17. Andersson T, Eliasson P, Aspenberg P. 2009. Tissue memory in healing tendons: short loading episodes stimulate healing. J Appl Physiol 107:417-21.

18. Palmes D, Spiegel HU, Schneider TO, et al. 2002. Achilles tendon healing: long-term biomechanical effects of postoperative mobilization and immobilization in a new mouse model. J Orthop Res 20:939-46.

19. Murrell GA, Lilly EG, 3rd, Goldner RD, et al. 1994. Effects of immobilization on Achilles tendon healing in a rat model. J Orthop Res 12:582-91.

20. Virchenko O, Aspenberg P. 2006. How can one platelet injection after tendon injury lead to a stronger tendon after 4 weeks? Interplay between early regeneration and mechanical stimulation. Acta Orthop 77:806-12.

21. Eliasson P, Andersson T, Aspenberg P. 2009. Rat Achilles tendon healing: mechanical loading and gene expression. J Appl Physiol 107:399-407.

22. Morey-Holton ER, Globus RK. 2002. Hindlimb unloading rodent model: technical aspects. J Appl Physiol 92:1367-77.

23. Miyatake S, Tohyama H, Kondo E, et al. 2008. Local administration of interleukin-1 receptor antagonist inhibits deterioration of mechanical properties of the stress-shielded patellar tendon. J Biomech 41:884-9. 
24. Magnusson SP, Hansen M, Langberg H, et al. 2007. The adaptability of tendon to loading differs in men and women. Int J Exp Pathol 88:237-40. 
Figure 1. Experimental set-up. Numbers represent the days 1-14 after surgery. The rats were allowed to run on a treadmill for 30 minutes per day, either day 2-5 or day 8-11. The early group was euthanized on day 8 , while the late group was euthanized on day 14. The two groups for histological evaluation were sacrificed on day 5, 1 hour after the last loading episode.

Figure 2. Peak force (A) and Peak stress (B) at 8 and 14 days after tendon transection with or without loading. Controls were completely unloaded tendons harvested at the same time as the exercise groups. Exercise consisted of 30 minutes of running on a treadmill, once a day for 4 days. Peakforce for the exercise groups differed from corresponding control groups at each time point $(p<0.01)$. Peak stress for the exercise group differed from the corresponding control group at the early time point $(p<0.05)$. 
Figure 3. Bleeding in the tendon callus. a) Overview of healing tendon tissue, black broken line indicates the interface between callus tissue and transected tendon stump (magnification $x 2$ ) and b) Area of bleeding, extravasated erythrocytes in the tissue (magnification $\times 40)$. 
Table 1. Results from the biomechanical evaluation

\begin{tabular}{l|llll|lll}
\hline & \multicolumn{3}{|c|}{ Early (8d) } & \multicolumn{3}{c}{ Late (14d) } \\
\hline Treatment & Control & Exercise & $95 \%$ CI & Control & Exercise & $95 \%$ CI \\
\hline $\mathrm{N}$ & 9 & 8 & & 10 & 9 \\
Peak force (N) & $7.3 \pm 2.8$ & $11 \pm 3.2$ & 15 to $100^{\mathrm{a}}$ & $12 \pm 2.4$ & $17 \pm 5.3$ & 16 to $83^{\mathrm{b}}$ \\
Peak stress (MPa) & $1.8 \pm 0.5$ & $2.6 \pm 0.8$ & 4 to $80^{\mathrm{a}}$ & $2.8 \pm 1.0$ & $3.9 \pm 1.3$ & -0.2 to $79^{\mathrm{c}}$ \\
Cross-sectional area (mm $\left.{ }^{2}\right)$ & $4.1 \pm 1.0$ & $4.5 \pm 0.6$ & -10 to 33 & $4.5 \pm 1.2$ & $4.7 \pm 1.2$ & -22 to 29 \\
Gap distance (mm) & $9.6 \pm 0.9$ & $9.7 \pm 1.0$ & -10 to 11 & $9.4 \pm 1.3$ & $9.1 \pm 1.1$ & -18 to 10 \\
Stiffness (N/mm) & $3.4 \pm 0.8$ & $4.5 \pm 1.2$ & 0.4 to $61^{\mathrm{a}}$ & $5.1 \pm 0.9$ & $6.5 \pm 1.3$ & 7 to $49^{\mathrm{b}}$ \\
Elastic modulus (MPa) & $12 \pm 3.7$ & $14 \pm 4.1$ & -16 to 51 & $16 \pm 6.2$ & $19 \pm 7.5$ & -23 to 60 \\
Energy (Nmm) & $11 \pm 6.1$ & $19 \pm 8.0$ & 9 to $141^{\mathrm{a}}$ & $19 \pm 7.5$ & $29 \pm 11$ & 6 to $99^{\mathrm{b}}$ \\
\hline
\end{tabular}

$95 \%$ CI means $95 \%$ confidence interval for comparison between the exercise group and unloaded controls

${ }^{a}$ significant difference from corresponding control group (d 8), $p<0.05$.

${ }^{b}$ significant difference from corresponding control group (d14), $p<0.05$.

${ }^{c} p=0.051$ 
Table 2. Results from the histological evaluation.

\begin{tabular}{|c|c|c|c|c|c|c|}
\hline \multirow[b]{2}{*}{ Sample ID } & \multicolumn{3}{|c|}{ Bleeding score } & \multicolumn{2}{|c|}{ Guessed treatment } & \multirow{2}{*}{$\begin{array}{c}\text { True } \\
\text { treatment }\end{array}$} \\
\hline & Investigator 1 & Investigator 2 & Sum & Investigator 1 & Investigator 2 & \\
\hline SA 246 & 0 & 0 & 0 & Control & Control & Control \\
\hline SA 247 & 1 & 1 & 2 & Exercise & Exercise & Exercise \\
\hline SA 248 & 0 & 0 & 0 & Control & Control & Control \\
\hline SA 249 & 1 & 1 & 2 & Exercise & Exercise & Exercise \\
\hline SA 250 & 0.5 & 1 & 1.5 & Control & Control & Control \\
\hline SA 251 & 1 & 1 & 2 & Exercise & Exercise & Exercise \\
\hline SA 252 & 0 & 0 & 0 & Control & Control & Control \\
\hline SA253 & 0 & 0 & 0 & Control & Control & Control \\
\hline SA254 & 1 & 1 & 2 & Exercise & Exercise & Exercise \\
\hline SA 255 & 1 & 0 & 1 & Exercise & Exercise & Exercise \\
\hline
\end{tabular}


Figure 1. Experiment set-up. Numbers represent the days 1-14 after surgery. The rats were allowed to run on a treadmill for 30 minutes per day, either day $2-5$ or day $8-11$. The early groups were sacrificed on day 8 while the late groups were sacrificed on day 14. The two groups for histological evaluation were sacrificed on day 5, 1 hour after finishing the final loading episode. $168 \times 52 \mathrm{~mm}(600 \times 600 \mathrm{DPI})$ 
a

\section{Peak force (N)}

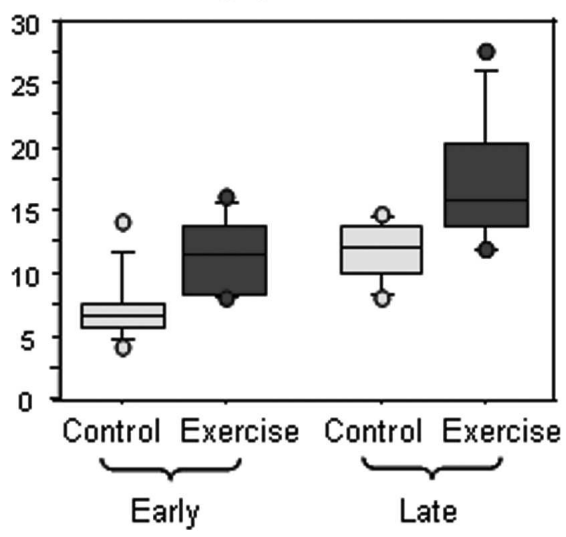

b

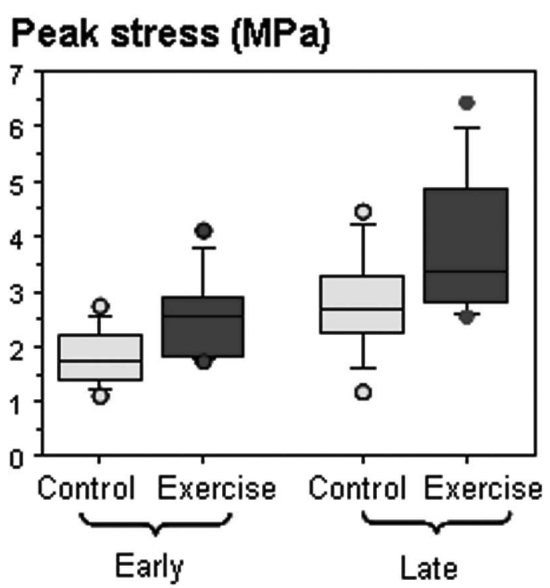

Figure 2. Peak force (A) and Peak stress (B) at 8 and 14 days after tendon transection with or without loading. Controls were completely unloaded tendons harvested at the same time as the exercise groups. Exercise consisted of 30 minutes of walking on a treadmill, once a day for 4 days. $82 \times 45 \mathrm{~mm}(600 \times 600$ DPI $)$ 
Figure 3. Bleeding in the tendon callus. a) Overview of healing tendon tissue, black broken line indicates the interface between callus tissue and transected tendon stump (magnification $\times 2$ ) and b) Area of bleeding, extravasated erythrocytes in the tissue (magnification $\times 40$ ).

$105 \times 56 \mathrm{~mm}(600 \times 600 \mathrm{DPI})$ 